$\mathrm{AB} 0164$

\section{THE EXPRESSION AND CLINICAL SIGNIFICANCE OF DIFFERENT FORMS OF LILRA3 IN SYSTEMIC LUPUS ERYTHEMATOSUS}

Yan Du, Meiju Zhou, Xinyu Wu, Jing Xue, Huaxiang Wu, Yujie Jiang. Zhejiang university school of medicine second affiliated hospital, Hangzhou, China

Background: LILRA3 is a member of the LILR family produced as a soluble molecule by monocytes and macrophages. A 6.7-kb deletion in the gene of LILRA3 results in a null allele and an absence of function. The frequencies of the $6.7-\mathrm{kb}$ deletion vary greatly in different populations, considerably higher in Northeastern Asians (0.56-0.84) compared with Africans (0.10) or Europeans (0.17). The homozygous LILRA3 deletion (nonfunctional LILRA3) has been demonstrated to be associated with Sjogren's syndrome (SS) and multiple sclerosis (MS) in Caucasians. However, in our previous studies, we found that in Chinese Han population, the LILRA3 non-deletion (namely functional LILRA3) contributes to susceptibility and subphenotypes of SLE and SS. With respect to the significant role of LILRA3 in immune-modulatory functions, we studied the role of LILRA3 in SLE.

Objectives: Our previous study has shown that functional LILRA3 contributes to susceptibility and subphenotypes of systemic lupus erythematosus (SLE). However, the mechanism remains unclear. We aimed to evaluate the role of LILRA3 in SLE.

Methods: 126 SLE patients and 48 healthy controls were recruited in this study. Functional studies were performed using intracellular flow cytometry and ELISA. Results: Both LILRA3 levels in serum and $\mathrm{CD} 14^{+}$monocytes were significantly elevated in SLE patients compared with healthy controls. Elevated LILRA3 level was found positively correlated with SLEDAI. Furthermore, more elevated LILRA3 levels were found in patients with higher SLEDAI, presence of lupus nephritis and thrombocytopenia.

Conclusion: Both LILRA3 levels in serum and CD14+ monocytes significantly increased in SLE and positively correlated with disease activity and severity. The up-regulation of LILRA3 expression may serve as a biomarker of disease activity and severity of SLE.

Table 1. Correlations of LILRA3 levels with the studied parameters in SLE patients

\begin{tabular}{lcccc}
\hline Clinical manifestations & \multicolumn{2}{c}{ LILRA3 in monocytes (MFI) } & \multicolumn{2}{c}{ LILRA3 in serum (pg/mL) } \\
& Spearman's $r$ & $P$ value & Spearman's $r$ & $P$ value \\
\hline SLEDAI & $\mathbf{0 . 3 9 8}$ & $\mathbf{0 . 0 0 1 8 ^ { * * }}$ & $\mathbf{0 . 2 5 7}$ & $\mathbf{0 . 0 0 3 6 ^ { * * }}$ \\
24h proteinuria excretion & $\mathbf{0 . 3 2 8}$ & $\mathbf{0 . 0 2 4 ^ { * }}$ & 0.159 & 0.09 \\
Leucocytes & -0.033 & 0.680 & -0.253 & $\mathbf{0 . 0 0 3 7 ^ { * * }}$ \\
Thrombocytes & -0.176 & 0.213 & -0.144 & 0.11 \\
Anti-dsDNA Ab & 0.066 & 0.627 & -0.061 & 0.498 \\
C3 & -0.138 & 0.290 & -0.076 & 0.392 \\
C4 & -0.140 & 0.282 & -0.025 & 0.777 \\
IgA & 0.068 & 0.604 & 0.017 & 0.852 \\
IgM & 0.107 & 0.417 & 0.053 & 0.557 \\
IgG & 0.143 & 0.218 & 0.228 & $0.01^{*}$ \\
CRP & -0.164 & 0.237 & 0.088 & 0.339 \\
\hline
\end{tabular}

SLE: systemic lupus erythematosus; SLEDAI: systemic lupus erythematosus disease activity index; LILRA3: Leukocyte immunoglobulin-like receptor A3; ANA: antinuclear antibody; AntidsDNA Ab: anti-double strand DNA Antibody; ACL: anticardiolipin antibody; AnuA:

antinucleosome antibody; Sm: anti-Smith antibody; SS: anti-SSA antibody; C3 Complement component 3; C4: Complement component 4. CRP: C-reactive protein. ${ }^{\star} P<0.05,{ }^{\star *} P<$

01. Spearman's correlation coefficient $(r)$ was applied to detect correlation between two types of numerical data.

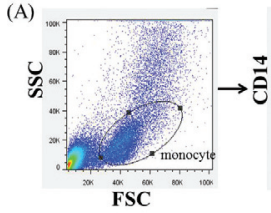

(B)

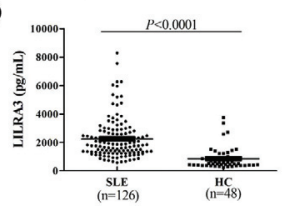

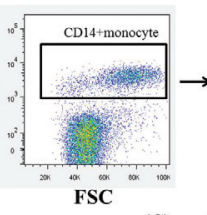

$(\mathrm{C})$

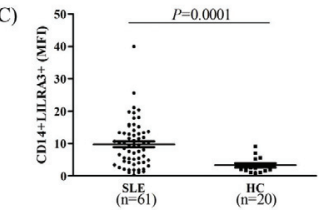

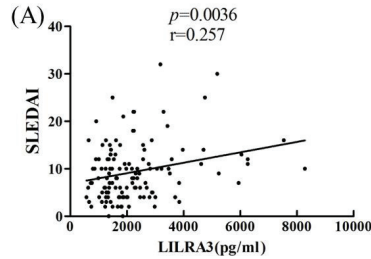
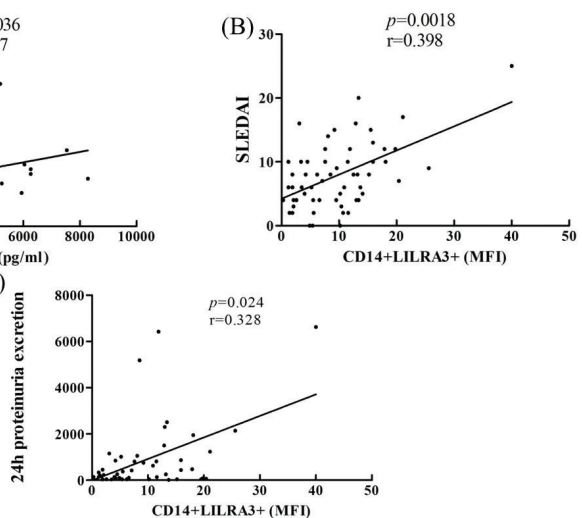

Figure 2
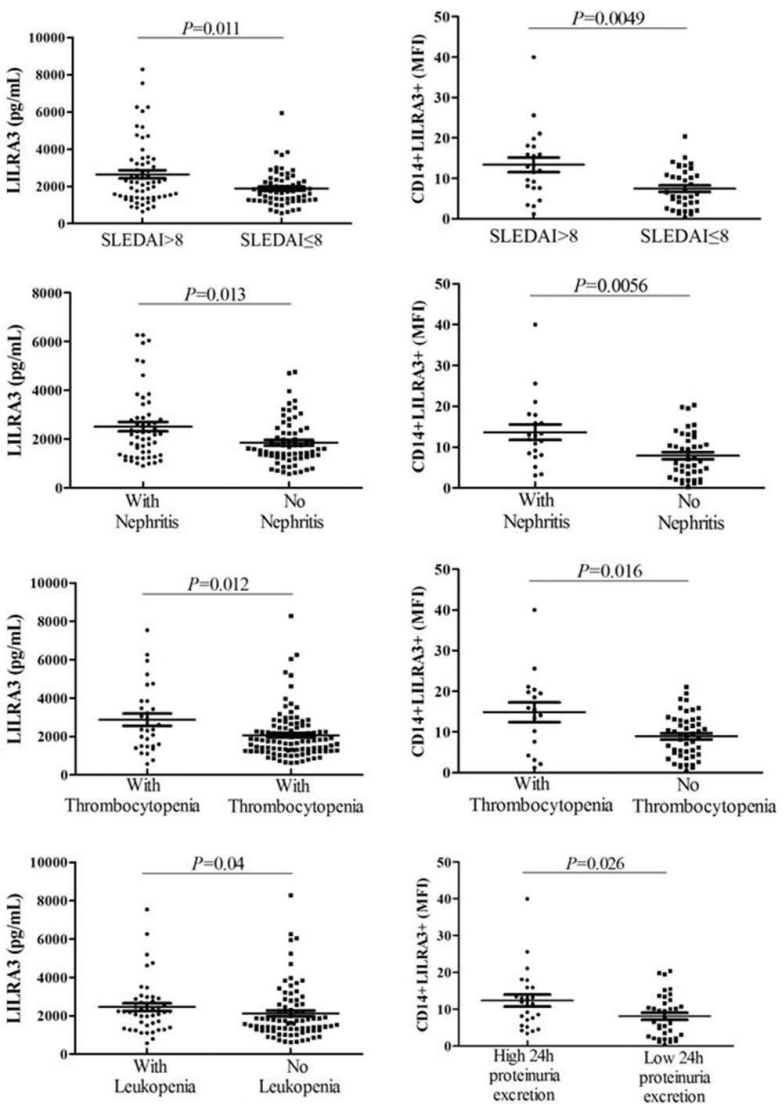

(A)

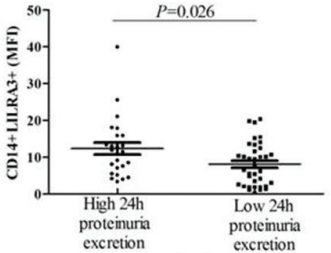

(B)
Figure 3

\section{REFERENCES}

[1] Du, Y., Y. Su, J. He, Y. Yang, Y. Shi, Y. Cui, C. Luo, X. Wu, X. Liu, F. Hu, X. Ma, L. Zheng, J. Zhang, X. Zuo, Y. Sheng, L. Wu, X. Zhang, J. Guo, and $\mathrm{Z}$. $\mathrm{Li}$, Impact of the leucocyte immunoglobulin-like receptor $\mathrm{A} 3$ (LILRA3) on susceptibility and subphenotypes of systemic lupus erythematosus and Sjogren's syndrome. Ann Rheum Dis, 2015. 74(11): p. 2070-5.

Disclosure of Interests: None declared

DOI: 10.1136/annrheumdis-2019-eular.2418 